\title{
Les enjeux du discours scientifique : la stratégie de véridiction
}

Jacky Martin

\section{CpenEdition}

\section{Journals}

Édition électronique

URL : http://journals.openedition.org/asp/3414

DOI : 10.4000/asp.3414

ISSN : 2108-6354

\section{Éditeur}

Groupe d'étude et de recherche en anglais de spécialité

\section{Édition imprimée}

Date de publication : 1 décembre 1996

Pagination : 13-31

ISSN : 1246-8185

\section{Référence électronique}

Jacky Martin, «Les enjeux du discours scientifique : la stratégie de véridiction », ASp [En ligne]

11-14 | 1996, mis en ligne le 05 avril 2013, consulté le 03 mai 2019. URL : http://

journals.openedition.org/asp/3414 ; DOI : 10.4000/asp.3414

Ce document a été généré automatiquement le 3 mai 2019.

Tous droits réservés 


\title{
Les enjeux du discours scientifique : la stratégie de véridiction
}

\author{
Jacky Martin
}

Cet article $^{1}$ se propose d'examiner le fonctionnement des stratégies textuelles à l'intérieur d'un article de recherche du domaine immunologique ${ }^{2}$ dans le but de proposer de nouvelles hypothèses pour l'analyse du discours scientifique (DS).

\section{Contexte de la présente recherche}

1.1.

2 Le paradigme dominant dans l'analyse du DS est celui qu'énoncent diversement, mais dans une certaine convergence, par des chercheurs comme Sager, Swales, Trimble ou Widdowson, pour ne citer que ceux-là. Il s'agit de l'orientation de recherche la plus productive et la plus féconde de ces dernières années. Pour essayer de la cerner en quelques mots (forcément réducteurs), nous nous en tiendrons à l'ouvrage le plus récent, celui de Swales (1990). L'idée centrale est que le DS est constitué d'un noyau central invariant («content schemata», 1990: 9-10) qui doit être adapté à divers contextes de communication par un certain nombre de techniques rhétoriques appropriées ( formal schemata» 1990 : 10). Ces techniques portent sur la mise en discours du propos du destinateur, mais surtout, sur l'étude des paramètres identifiables dans le contexte de réception et des types de configurations textuelles ("Text-types») auxquelles ces paramètres donnent lieu. Ils mettent en jeu trois concepts corrélés : la « communauté de discours", la notion de "genre» et celle de "tâche». Ces orientations, maintenant largement diffusées, ont donné une impulsion décisive à la recherche sur le DS et à sa didactisation.

1.2.

3 Il semble pourtant que, sous l'influence des travaux déjà bien connus des sociologues de la science ${ }^{3}$, il faille, sinon remettre en cause, du moins réviser ou compléter ce qu'il faut bien appeler aussi un paradigme. Ces chercheurs ont fait voler en éclats le paradigme Mertonien (qui semble encore sous-tendre les travaux de «néo-rhétoriciens » comme 
Swales) posant la science comme la clarification progressive et consensuelle d'un discours de vérité sur la réalité dont la traduction en discours ne pose ensuite que des problèmes d'adaptation au contexte choisi. On doit l'impulsion initiale aux travaux fondateurs de chercheurs "transversaux » comme T. Kuhn et P. Feyerabend qui ont dénoncé l'idée communément reçue d'une marche ininterrompue de la science vers l'établissement de la vérité pour la remplacer par la notion de paradigmes successifs séparés par des crises de transition. À partir des années 1970, tout un ensemble de recherches a été conduit, non plus sur les périphéries de l'activité scientifique, mais au sein même des laboratoires les plus prestigieux des États-Unis et d'ailleurs. En sont ressorties les notions maintenant incontournables de "construction des faits scientifiques" à partir d'un ensemble de transcriptions «littéraires» du donné référentiel, dans le cadre d'une communauté scientifique pour laquelle la publication de travaux coïncide avec l'acquisition de notoriété, immédiatement reconvertie en moyens matériels et influence ${ }^{4}$.

1.3 .

4 Il est temps d'esquisser une autre conception du DS que celle, dite de la «windowpane illusion » c'est-à-dire d'un discours tentant, avec plus ou moins de succès, de laisser transparaître une vérité scientifique qui cernerait de plus en plus près la réalité qui nous entoure.

5 1.3.1. Dans la véridiction (faisant référence à ce qui est « véridique » et non plus à ce qui est « vrai »), le discours ne saurait être envisagé, au moins en tant que tel, dans l'optique d'une quelconque objectivité, neutralité ou impartialité qui ne peuvent, dès lors, être considérés que comme des "images » désignant l'« enrobage idéologique» du discours scientifique, sa motivation aux yeux des membres de la communauté scientifique et sa justification pour l'ensemble de la société qui, de près ou de loin, la finance.

6 1.3.2. Dans cette optique, les scientifiques disparaissent en tant qu'individus producteurs de sens et se trouvent réintégrés en tant que collaborateurs à la rédaction d'un texte commun, collectivement accrédité, correspondant au paradigme d'une discipline donnée. Le paradigme est l'ensemble de ce qui est consensuellement reconnu comme dicible concernant un certain segment du réel. Ce segment du réel est lui-même construit par le discours s'y rapportant et donc va se trouver redéfini et contesté à chaque changement de paradigme.

7 1.3.3. Le DS est donc fondamentalement partisan et agonistique : il s'agit, pour chaque communauté paradigmatique, de renforcer et d'étendre le champ de validité du discours qui la fonde et de neutraliser les discours concurrents jusqu'au moment où se produisent les « crises » décrites par Kuhn. Ces crises précèdent les changements de paradigme, ce qui signifie pour nous, des changements d'univers de discours.

8 1.3.4. Le DS n'a pas pour vocation première de mettre en œuvre des techniques de communication de façon à faire passer un contenu informationnel. Si l'on voulait cultiver le paradoxe, on pourrait dire, au contraire, qu'il oscille entre deux extrêmes de la noncommunication: l'ésotérisme fortement ressenti par le non-initié à la discipline et l'hyperspécialisation dans laquelle le sens des rapports et les visions d'ensemble sont parfois difficiles à reconstruire. Le texte scientifique n'est pas produit pour être compris de tous mais pour prendre sa place dans le "grand texte» disciplinaire. Ce sont les sutures avec ce grand texte qui sont importantes plutôt que l'accroche avec un public. Il ne s'adresse donc qu'à un public déjà informé qui souhaite étendre des connaissances déjà acquises. Par opposition, les stratégies de communication (trop souvent confondues avec 
le texte scientifique proprement dit) visent à rendre accessibles des connaissances déjà formées pour des destinataires qui, pour différentes motivations, souhaitent les acquérir. Cette orientation va à l'encontre des finalités du DS dans la mesure où l'article scientifique s'adresse à un public déjà initié qui s'attend plus à recevoir des preuves de la validité du contenu informationnel de l'article que des assurances sur sa nouveauté, dans la mesure où le texte révélé est une extrapolation par rapport à un discours déjà produit. C'est moins la nouveauté et l'importance du contenu de discours qui est en cause que sa recevabilité et sa fonctionnalité à l'intérieur d'un paradigme.

1.4.

9 Telles sont les notions qui forment le soubassement de cette recherche. Même si je ne prétends pas obtenir l'assentiment de tous nos lecteurs, elles ont du moins le mérite de mettre en évidence les enjeux actuels de la recherche dans le domaine de la linguistique du DS. Il est clair que je n'ai fait que reprendre les idées formulées par les sociologues de la science. Pourtant, il m'a paru nécessaire d'apporter un certain nombre d'adaptations pour tester la validité de leurs idées dans le domaine de la linguistique du discours.

10 1.4.1. Il fallait d'abord contester le primat donné aux déterminations socio-économiques par certains sociologues de la science ${ }^{5}$. Même si, pour Latour, le discours n'est pas directement déterminé de l'« extérieur » par un hors-texte, mais se trouve pris dans un " réseau » de circonstants qui, par leur interaction créent un événement sur le plan scientifique, il est clair qu'il n'occupe pas la place centrale que nous lui donnons. Même si le DS est soumis à la double contrainte de sa justification sur le plan socio-économique (mise en évidence par les sociologues de la science) et de sa vérification sur le plan expérimental (toujours mise en avant par les scientifiques eux-mêmes), nous posons que c'est en tant que discours, c'est-à-dire dans l'échange et la négociation des textes à l'intérieur de la communauté scientifique, qu'il est d'abord validé. Cette position ne revient pas à nier l'incidence nécessaire de ces discours sur leur milieu, ni de ce dernier sur leur propre définition, mais à placer cette incidence dans un retrait par rapport au référentiel. C'est ce retrait qui caractérise les discours de savoir par rapport aux discours de pratique, dans la mesure où ils sont d'abord définis de façon interne et ensuite rapportés aux situations auxquelles ils peuvent faire référence et non l'inverse. Tout énoncé de la "vie courante », au contraire, se rapporte d'abord à une situation précise et peut, ensuite (mais non obligatoirement) être rapporté à une formation discursive (tendance, précepte, principe, système, etc.).

11 1.4.2. Il m'a semblé par ailleurs que, dès l'instant où l'on marquait ce palier de définition au niveau du discours, apparaissaient beaucoup plus clairement les différentes facettes du fonctionnement agonistique du DS. Le texte scientifique nous fait l'effet -- pour faire simple et clair -- d'un champ clos dans lequel les forces de la méthode affrontent celles de la dissociation. Plus exactement, puisque la victoire n'est pas concevable dans l'un ou l'autre camp, le texte scientifique est un point d'équilibre dans une situation référentielle en constante déstabilisation. Certes, cet aspect agonistique n'a pas échappé aux sociologues de la science ${ }^{6}$ mais ils ont surtout montré l'importance de deux «fronts " stratégiques: la validation qui place le texte scientifique en équilibre précaire entre ressassement et déviation par rapport au paradigme, et la facticité qui le montre en train de façonner un objet de discours à mi-chemin entre le déjà connu, ou objet invisible, et l'artefact, ou faux objet. J'ajoute, ou précise, deux autres « fronts » qui seront étudiés plus sommairement dans l'analyse qui suit ce préambule : l'enjeu de la logification, c'est à dire tout ce qui dans le texte concourt à éliminer l'irrationnel, le fortuit, ou le 
désordonné, et l'enjeu de la mesure par laquelle le texte scientifique essaye de se situer à mi-chemin entre l'unique (non reproductible), et l'aléatoire (ou incommensurable). Tout dans le DS est affaire de tensions et d'équilibres. On est loin d'une conception du DS comme froide et rigoureuse transcription de la vérité scientifique.

1.4.3. Il m'a semblé également que le concept de «construction» du DS, même si ses auteurs s'en défendent, présupposait trop fortement l'idée que le discours se "bâtit » à partir de matériaux plus ou moins pré-construits en fonction d'un certain projet. Une telle conception s'opposait à notre travail sur corpora de textes scientifiques qui faisaient apparaitre que, même si le discours était travaillé au plus profond par les «enjeux » que nous avons décrits précédemment, ce travail d'engendrement ne s'en exerçait pas moins à partir d'une structure textuelle stable. Cet acquis textuel, qui relève pour certains du lexique de spécialité, constitue selon moi un pré-construit discursif auquel j'ai donné le nom de macrotexte. Pour penser cette articulation entre du fixe et du variable à l'intérieur du DS, j'ai eu recours au concept de structure métastable tel que le définit M.A.K. Halliday (1994).

1.4.4. Finalement, il m'a semblé que les sociologues de la science faisaient assez peu de cas de ce qui, pour nous médiateurs du texte scientifique, était de la plus grande importance, à savoir la dissémination du DS. Non seulement nous sommes amenés à intervenir dans la définition et la mise en conformité de l'article de recherche, mais nous intervenons aussi à toutes les étapes de sa diffusion. Il m'a semblé judicieux de concevoir et décrire les stratégies spécifiques qui règlent ce processus, en tenant compte du fait qu'il s'agit de stratégies secondes. La didactisation et la vulgarisation du savoir (qui, du point de vue textuel, n'ont presque rien à voir l'une avec l'autre) se fondent sur un texte déjà constitué (le texte paradigmatique) pour le recentrer en fonction de préoccupations strictement communicatives. Plus avant, il était important de penser l'étape ultime dans la dissémination du DS: sa dénaturation à travers les utilisations idéologiques et la consommation que notre société en fait massivement. S'agit-il alors toujours d'un DS reconnaissable (rapportable au paradigme d'origine) ou d'un tout autre texte? Comment s'effectue le "délitement » du DS au contact de valeurs extrinsèques? Quelles sont les notions scientifiques filtrées par nos sociétés? Ces questions, et bien d'autres, sont loin d'être uniquement rhétoriques.

\section{Formulation de nouveaux concepts}

\section{1.}

Pour concrétiser ma conception du DS comme structure métastable, j'ai donc posé le concept de macrotexte qui se situe à mi-chemin entre la terminologie et le discours proprement dit. Il s'agit d'un niveau textuel pré-construit formé non seulement de termes mais de chaînes syntaxiques pré-assertives qui conditionnent la compréhension et la production du DS dans un domaine donné. Pour essayer d'y voir clair, si l'on comparait les termes aux pièces d'un jeu d'échecs, le macrotexte serait les « gambits » ou tactiques déjà répertoriées qui, elles-mêmes, vont s'intégrer dans les parties effectivement jouées, dans notre analogie, les textes produits.

2.2.

La dynamique du DS est liée aux stratégies sans lesquelles le macrotexte ne nous serait pas accessible. Nous avons affaire à deux niveaux d'engendrement du DS (un peu dans le 
même rapport que "structure profonde » et «structure de surface» dans la topique chomskienne), et non à deux niveaux superposés de construction. Les stratégies sont de deux ordres, celle que nous qualifions d'interne, la stratégie de véridiction qui vise à étendre et diversifier le macrotexte, et celles, externes, qui visent à le disséminer dans la société : il s'agit des stratégies de communication («Outsider strategies ») : transmission du savoir et vulgarisation des connaissances et des stratégies d'appropriation («Extraneous strategies ») comme la publicité par exemple ou l'utilisation de divers DS pour des causes humanitaires. Si, dans le cas des stratégies de communication, le macrotexte est utilisé de façon quasiment tautologique, il est en revanche asservi à des finalités idéologiques dans les stratégies d'appropriation.

\section{Objet de l'étude}

16 Je me propose d'analyser concrètement, à propos d'un article de Science dans le domaine de l'immunologie, certains aspects de la stratégie de véridiction, particulièrement sous l'angle de la validation et de la facticité, plus sommairement, du point de vue de la logification et de la mesure. Je tirerai ensuite quelques conclusions sur l'orientation de la recherche dans le domaine de la linguistique du DS.

\section{La stratégie de véridiction}

Chacun des "enjeux» décrits précédemment doit être conçu comme les torons individuellement isolables d'une corde, mais dont le tressage produit l'article de recherche. Ce tressage pourra faire apparaître, de façon plus ou moins appuyée, selon la finalité de l'article ou la nature du sujet abordé (article de recherche, article de synthèse, demande de subvention) l'un ou l'autre des « enjeux » définis précédemment. On est loin, par conséquent, du schéma canonique IMRAD qui, sans être invalidé, se trouve recreusé par les dynamiques profondes que nous avons mises en évidence.

4.1.

En ce qui concerne l'article de recherche qui nous occupe, la validation consiste d'abord à établir l'accréditation des rédacteurs de l'article, c'est-à-dire le « droit de contribuer au paradigme. » Ce plan textuel définit le statut des producteurs de textes (par opposition à la notion d'auteur liée à l'identité distinctive et originale du scripteur) dans le cadre de la communauté scientifique. Elle se traduit ensuite par la validation proprement dite du texte de l'article, c'est-à-dire sa recevabilité au sein du paradigme.

19 4.1.1. L'accréditation commence bien avant la publication de l'article à toutes les étapes nombreuses de sa gestation. On sait en effet qu'il doit être soumis aux différentes instances de décision concernant sa recevabilité : à l'intérieur du laboratoire (rôle des conversations informelles entre chercheurs et contrôle permanent du responsable $\mathrm{du}$ laboratoire); au niveau inter-laboratoire, comme ce fut le cas dans notre article qui rassemble les contributions convergentes de plusieurs établissements de recherche; et surtout, dans le va-et-vient qui s'établit entre les auteurs de l'article et les instances de contrôle de la publication: arbitres (referees). Le DS est un discours, contraint mais consensuel, issu de la négociation, dans lequel la notion d'auteur cède la place à celle d'« autorisé » à produire un texte. Comme l'a justement fait remarquer Thomas Miller 
(1993 : 56), la distinction entre auteur et lecteur tend également à s'estomper pour laisser la place à un concept (à définir) de « scientifiquement recevable ».

Diverses traces de ces échanges subsistent parfois: dans le cadre de l'article choisi pour cette analyse, dans l'indication du décalage entre la date d'écriture et la date de publication dans Science, en fin d'article. Vont aussi fonctionner comme " accréditifs », la mention des diverses dotations financières, subventions de recherche ou autres cautions officielles accordées par le milieu industriel ou professionnel ${ }^{7}$. Iront également dans le même sens, les indications concernant les divers centres de recherche qui ont été impliqués, de près ou de loin, dans cette recherche ${ }^{8}$ ainsi que l'énoncé de leurs rôles et types de participation respectifs.

Bien sûr, la caution déterminante vient de la publication de cet article dans une revue aussi prestigieuse que Science, dont on connaît la longue liste d'attente et donc la sévérité du comité de lecture. Doit être également présent à l'esprit, le statut généraliste de cet organe de publication, et donc le caractère assez exceptionnel de ses publications dans le domaine scientifique.

Participeront également de cette accréditation du contenu de l'article sa conformité exacte avec le format (style sheet) de la revue, sa présentation, sa longueur et la place des encarts non-textuels de même que le style de sa bibliographie. On remarquera qu'en l'occurrence le sacro-saint schéma IMRAD n'est pas non plus respecté ici, en conformité avec la politique éditoriale de la revue : se trouve renvoyé en notes ce qui correspondrait à la section Materials and Methods dans ledit schéma.

Il faut également ajouter que la partie éditoriale de la revue (non reproduite) met clairement en évidence, dans la présentation des articles publiés, aussi bien l'intérêt scientifique que les zones d'obscurité de cette publication, relançant, du même coup, le processus de déstabilisation contre lequel l'article dans son ensemble semblait s'être ligué.

24 4.1.2 La validation proprement dite concerne la fiabilité du contenu de l'article, qui ne peut être reçu que lorsque ses producteurs ont été, au préalable, dûment accrédités. La validation établit la conformité de l'article par rapport au macrotexte et, plus directement, définit les conditions de son acceptation par la communauté scientifique concernée. Notons au passage que ce n'est qu'exceptionnellement que cette validation s'effectue de façon directement polémique : cet aspect, fondamental dans le renforcement des paradigmes, ne manquera pas néanmoins d'être représenté, par exemple, sous la forme d'une omission dans la liste des cautions scientifiques citées en référence, et plus directement encore, dans le choix stratégique des "parrains " scientifiques de l'article ${ }^{9}$. L'essentiel des fonctions rhétoriques de validation vise à établir la continuité entre le macrotexte et le texte de l'article. Ce « raccordement/suture » avec le macrotexte se fait selon trois relations distinctes.

4.1.2.1 La relation citationnelle qui fait que des pans entiers du macrotexte sont simplement désignés par des références bibliographiques et donc solidement posés comme non susceptibles d'être remis en cause : il s'agit de la « doxa » à laquelle il est fait seulement référence sans discussion. Notons qu'il ne s'agit pas, comme dans le texte littéraire par exemple, de références «flatteuses » destinées à venir conforter ce qui est énoncé dans le corps de l'article mais bel et bien d'un fragment du macrotexte paradigmatique, lequel n'a plus lieu d'être développé, ni commenté, et qui pourra être évoqué plusieurs fois dans le corps de l'article sans être physiquement présent. Il s'agit de 
son fondement stratégique sur lequel tout le reste va être bâti. Le choix de ces références bibliographiques est loin d'être purement académique: il participe d'une stratégie complexe de sélection des parrainages de l'article. À ce niveau, il n'y a donc aucune différence entre validation et accréditation, dans la mesure où citer ses références revient à revendiquer ses cautions scientifiques.

4.1.2.2. La relation implicite, par contre, fait référence à un texte déplacé ou enfoui, qui cautionnerait des éléments comme ceux présents au début du texte: « V » et « $C$ domains » eux-mêmes subdivisés en $\mathrm{Vl}$ et Vh par exemple (notons au passage que la siglaison est à considérer comme une étape intermédiaire entre la citation explicite du macrotexte et son implicitation). Impossible de faire sens à partir de ces unités lexicales si l'on ne connaît pas à la fois leur référent et les rapports qu'elles entretiennent avec les autres à l'intérieur du macrotexte. Il ne s'agit plus comme précédemment de faire référence à des "hommes-textes", mais à un texte préexistant, texte-source qui seul donne sens aux unités lexicales sur lesquelles se fonde notre article. C'est cette connaissance du macrotexte, considérée comme acquise dans le lectorat de l'article, qui détermine sa validité, c'est à dire à la fois sa compréhension et sa recevabilité. Encore une fois, difficile de dissocier validation et accréditation, dans la mesure où les scientifiques ne sont pas des auteurs à proprement parler, mais des « hommes-textes » dont le nom est parfois indissociable d'un fragment du macrotexte. Ainsi, certaines techniques de production ou d'observation sont parfois seulement désignées par le nom de leur inventeur. Cette référence implicite contribue à déterminer une communauté de savoir, plus ou moins large, plus ou moins exclusive, selon le type et l'étendue des zones de savoir implicitées ${ }^{10}$.

4.1.2.3 La relation explicite : À différents moments stratégiques de l'article, on trouve des citations directes, ou même des emprunts à d'autres articles, provenant directement du macrotexte, qui jouent presque le rôle dévolu - si vous me passez l'incongruité de l'image - aux porte-greffe en chirurgie. Ces supports biologiques doivent être sains et de nature à favoriser la repousse des tissus ou organes greffés. Cette fonction d'acclimatation du texte " greffé » est évidente dans le paragraphe introductif de l'article ${ }^{11}$. Cette citation du macrotexte immunologique, qui va du début de l'article jusqu'à : «We now report..., » va servir de support à l'apport de texte nouveau. Notons à cette occasion que le macrotexte, en tant que structure «métastable, » est donc essentiellement déformable et adaptable, toujours reconnaissable en dépit des transformations auquel elle est soumise.

Ainsi, en relation avec la première phrase, il a été possible de déterminer (par l'étude des concordances portant sur de très nombreux textes du corpus immunologique) que la relation entre les différents termes et leurs sous-composantes : « antigen » et « recognition » ou « T cell » et « receptor, » est stable au niveau macrotextuel, c'est-à-dire qu'il n'est pas possible d'envisager d'autres combinaisons que <T Cells+recognize> (et non « identify, » ou "distinguish, " par exemple) et <T cell+receptor $>12$ (et non « identifier » ou "reactant »), mais que ces termes peuvent être positionnés, grammaticalement, les uns par rapport aux autres, dans un grand nombre de combinaisons en fonction des visées du texte : ainsi, " recognition, " «recognize, " « recognized» marqueront les différentes modalités d'un processus qui, tout en étant infléchi, demeure macrotextuellement stable. C'est ainsi que la structure du macrotexte perdure à travers ses transformations : Halliday va même plus loin en affirmant qu'elle ne survit que dans ses transformations.

Les transformations sont motivées, dans le cas présent, par la nécessité de mettre en relief le thème de l'article par une focalisation progressive des éléments du macrotexte 
sur lesquels va porter l'étude, c'est-à-dire le rôle et la structure des " $T$ cell receptors. » On voit bien, dans ces conditions, qu'il est vain, par exemple, de souligner sur le seul plan linguistique (parfois trop lourdement par des moyens statistiques), la récurrence de tournures passives comme "mediated by" et "composed of, " dont la fonction est différente sous l'apparente similarité de forme, mais qui concourent également à présenter le thème de l'article.

4.2

Dans la suite de l'article - à ses points d'articulation stratégiques - le macrotexte explicite va également réapparaître en combinaison avec des apports de texte affectés de diverses modalisations ${ }^{13}$. Ces phraséologies vont déterminer le degré d'acceptabilité de l'insertion du nouveau texte dans le macrotexte déjà reconnu. Ainsi, la seconde insertion (§ 6) équivaut à une quasi-certitude puisqu'elle désigne les résultats comme «compatible with models, » à la réserve près évoquée dans l'énoncé de conclusion : « probably precludes, » qui modalise légèrement l'assertion précédente.

Les autres inclusions de texte paradigmatique vont être placées sous le régime de modalisations de plus en plus insistantes: (cf. § 7) : «could explain », « may modulate », « The possibility that...may be implicated...has been raised ", en opposition avec « indeed » et " furthermore » qui font référence à un régime textuel beaucoup plus assertif. Nous découvrons, semble-t-il, les deux rythmes fondamentaux de la validation dans les textes scientifiques.

32 La création autour du macrotexte de référence de zones textuelles plus ou moins scientifiquement recevables moyennant certaines précautions modales savamment graduées. Il ne s'agit pas là de zones d'incertitude comme le pensent certains chercheurs, mais de « franges » à explorer susceptibles, à terme, de faire partie (ou de finalement être exclues) du macrotexte. C'est probablement dans la définition experte de ces franges que s'établissent les différences culturelles entre anglophones et non-anglophones et, par voie de conséquence, l'acceptabilité des articles scientifiques auprès des comités de lecture anglo-saxons.

La combinaison entre énoncés modalisés et énoncés fortement assertifs (qui n'ont pas reçu toute l'attention qu'ils méritent) correspond donc à deux types de validation. Cette désignation exclut l'idée d'une dichotomie entre acquis de recherche, non modalisés, et divers degrés d'incertitude caractérisés par des hedges qui jouent, dans l'esprit de leurs descripteurs, le rôle de bémols par rapport au degré de certitude maximale. Il apparaît, d'ailleurs, que les modalités, loin d'affaiblir l'assertion des "valeurs macrotextuelles ajoutées » auxquelles elles s'appliquent, ne font, au contraire, que renforcer leur validité. Une telle opposition entre assertivité et modalisation se trouve largement illustrée en conclusion de l'article. On y trouve ( $\$ 11$ ), un développement dans l'ensemble assertorique en strict vis-à-vis avec une conclusion purement prospective et très largement modalisée.

Il faut donc poser, au niveau de la validation, que le texte se construit sur trois types de rapports au macrotexte, qui se présentent chacun sous la forme d'une opposition dynamique: entre un texte cité et un texte occulté; entre un texte enfoui et un texte explicite ; entre un régime assertif et un régime modalisé. 


\section{La facticité} a d'engendrement fondamentaux $\mathrm{du}$ discours scientifique, l'analogie. Par ailleurs, l'analogie dont il est question ici, c'est-à-dire le rapport qu'établit l'article entre ce qui existe déjà dans le macrotexte - en l'occurrence, la description de la structure des anticorps - et ce qui est visé par le corps de l'article - la structure d'une « B chain of a T cell antigen receptor » - montre bien que la «facticité » de l'objet visé tient tout autant à sa correspondance avec un certain nombre d'observations («data») qu'à son bon accord avec le macrotexte paradigmatique. Il est crucial d'observer que le «fait scientifique » se construit au point de rencontre de ce double faisceau de preuves de nature purement discursives ${ }^{15}$. La structure des récepteurs des cellules $T$, telle qu'elle apparaît dans les résultats d'observation à nous communiqués dans les tableaux et photographies, est déduite, et donc " construite ", à partir d'une similarité postulée avec celle des anticorps. Il est permis également de penser, tant la structure du fait scientifique est liée au paradigme textuel, que les observations qui ont été faites l'ont été dans la perspective d'une éventuelle mise en parallèle entre les deux structures (cf. Table 1), et que ce parallèle, une fois posé, a dicté le choix des angles et le réglage des prises de vues ainsi que les codes de coloration dans les figures 1,2 et 3 . Indépendamment du cas particulier mais significatif que constitue ce texte, il faudra toujours apprécier, sur le plan de la facticité, la part respective de l'induction (interprétation des données à partir du macrotexte) et de l'extrapolation(prédiction à partir du macrotexte). Les deux orientations sont, en définitive, deux façons convergentes de lire le macrotexte face aux réalités référentielles.

La facticité (qui correspond à ce que Gross [1990] appelle "Presence») se réalise textuellement à trois niveaux.

5.1.

Dans l'externalisation du fait scientifique par des moyens linguistiques qui consistent à gommer toutes références au contexte d'énonciation : ainsi, les marques circonstancielles faisant référence à la situation réelle dans le laboratoire sont remplacées, comme on le verra plus bas, par l'énoncé des conditions objectives de production: le fait scientifique est essentiellement universel. Effacement, également, de toutes relations à ses « inventeurs » : nous avons ici une unique référence occasionnelle au «we » dont il faut apprécier non tellement l'ambiguïté que la « catholicité » : il s'agit en effet tout à la fois de la référence à un collectif (les membres de l'équipe), à un sous-groupe à l'intérieur de la communauté scientifique ainsi qu'à la communauté scientifique dans son ensemble.

L'essentiel de l'effet de généralisation du fait scientifique est produit par les phénomènes d'animation des inanimés (phénomènes encore mal étudiés) tels que, par exemple, dans le $\$ 1:$ « antibodies generally recognize antigens...TCRs interact with...which stimulate, etc. ». Plus 
que des phénomènes d'animation, qui n'ont pas tellement de sens dans le DS (ou qui doivent être rapportés aux métaphores latentes toujours présentes dans le DS), il faut y voir des jeux d'orientation des prédicats et des relations d'ordre dans la séquence des opérations dans lesquels les notions d'agent ou d'objet n'ont plus cours, mais où seules comptent les notions d'opérations et d'objets sur lesquels portent ces opérations.

5.2.

Le second facteur de facticité est lié à la vérifiabilité du fait scientifique. Tel qu'il nous est présenté dans ce texte, l'objet dont il est question a été produit en trois temps correspondant aux descriptifs contenus dans les notes 6,7 et 8 . Notons que ces indications de production - selon une tendance qui se confirme dans l'article de recherche contemporain - se trouvent exclues du corps de l'article et réduites au rang de notes de fin de texte. Apparaîtra d'autant plus clairement, en raison de la compression auxquelles elles ont été soumises, la structure grammaticale massivement passive de ces notes: noter que si la passivation est en quoi que ce soit impliquée dans le DS, elle concerne essentiellement cet aspect de la stratégie de véridiction et non l'ensemble de l'article de recherche.

41 Dans d'autres articles, c'est le processus de vérification qui sera massivement mis en relief, bien qu'il ne faille jamais perdre de vue le cercle vicieux - mis en évidence par Latour - qui se tisse entre conditions de vérification et conditions d'observation :

We have argued that accounts of the emergence of a new finding...entail a two-fold process of transformation. On the one hand, the analogical path is often replaced by a logical connection. On the other hand, the complex set of local circumstances which temporarily makes possible a weak link gives way to flashes of intuition. (1986 [1979]: 174)

En d'autres termes, on ne vérifie que ce qui a été expérimentalement reconnu comme pertinent et on ne confirme donc que ce qui a été posé en hypothèse.

5.3.

43 La troisième stratégie de facticité concerne la réification du fait scientifique. Il s'agit de le présenter tel qu'il apparaît dans sa nature fondamentale et non circonstancielle. C'est là qu'intervient idéalement la mathématisation - présente, dans notre article seulement en fonction d'appui au niveau des conditions d'observation -, mais également et surtout, les visualisations. Il sera utile, à ce propos, de redéfinir les valeurs respectives du textuel et du non-textuel dans le DS : en gros, il faut exclure la relation habituelle d'illustration, pour envisager une structure de complémentarité entre visuel et textuel. Cette structure est particulièrement claire dans notre article où le textuel souligne les éléments remarquables dans la représentation des alignements d'acides aminés mais se situe en retrait par rapport à la complexité des phénomènes désignés par les figures $(1,2$ et 3$)$, malheureusement mal reproduites dans notre photocopie. Impossible désormais de n'envisager que le contenu linguistique de l'article scientifique sans ses insertions de matériaux non-textuels : que serait une flore sans ses illustrations?

Il faut également remarquer que cette fonction de réification ne consiste pas simplement à montrer mais aussi et surtout à "apprêter " les référents en les simplifiant, en soulignant leurs aspects significatifs (code de couleurs), et en choisissant les angles et configurations sous lesquels ils ont été représentés. Alors que les critères habituels de réussite pour l'illustration d'un texte informatif sont l'authenticité et la spécificité, la représentation en DS porte plutôt sur la stabilité et la représentativité des données offertes aux lecteurs. 
Il faut finalement citer dans la fonction de réification le rôle déterminant (déjà souligné par Gusfield, 1976) que joue le résumé («Abstract») de l'article, lequel tend à faire accepter le fait paradoxal que l'article de recherche possède à la fois un contenu détachable du corps de l'article et qu'il est indissociable des chaînes explicatives qu'il contient. Il nous parait hasardeux dans ces conditions de dissocier, pour des raisons de commodité évidente, le résumé du corps de l'article, lesquels, dans notre optique, sont dans un rapport de tension: l'un n'existant pas sans l'autre, l'un désignant toujours l'autre, et réciproquement.

\section{La logification}

Le troisième "enjeu» qui se «livre" dans le texte concerne sa logification. Il est indissociable des deux précédents. Dans la mesure où la validation avère la recevabilité du fait scientifique et la facticité son existence réelle du point de vue du paradigme, la logification démontre, d'une part, l'inévitabilité de son existence : on ne peut confondre ce texte avec aucun autre et il est infiniment reproductible, et d'autre part, la logique de sa structure ou/et de son fonctionnement. ${ }^{16}$ Dans la mesure où il est licite (validation) de poser des objets (facticité), il est indispensable de tisser des fonctionnements de nature à les relier. Il s'agit, en l'occurrence, d'éliminer tout ce qui est simplement événementiel, seulement local ou erratique pour tracer entre les objets des "pathing devices» ou " sequences" (cf. Woolgar [1981], mais aussi Gusfield 1976) qui les relient de façon nécessaire. C'est, je crois, l'aspect du DS qui a été le mieux étudié.

Notre texte, de ce point de vue se présente, globalement, comme une structure comparative, longuement argumentée, débouchant sur des déductions et des extrapolations. Je crois qu'il est possible, même à ce niveau très superficiel de l'analyse de la logification, de voir en quoi notre approche s'apparente à celle des néo-rhétoriciens et, dans quelle mesure aussi, elle s'en démarque: ce qui, pour les néo-rhétoriciens, correspond à des formes rhétoriques dont il convient de donner simplement l'inventaire et la combinatoire, se trouve dans notre optique construit dans le cadre d'une stratégie globale de production de sens. La logification doit être saisie comme la résultante textuelle d'un couple de forces divergentes: avec d'une part, le doute et le scepticisme latent de la communauté scientifique concernant l'aboutissement de recherches qui ont occupé de nombreux laboratoires dans le monde ${ }^{17}$ et, d'autre part, l'angoisse de faire jouer trop à fond le paradigme et l'extrapolation pour caractériser l'objet de recherche. Si l'on garde en mémoire ces deux "abîmes" de non-sens entre lesquels sinue l'argumentation $\mathrm{du}$ texte, on pourra non seulement caractériser les trames argumentatives (non seulement les «figures ») du texte mais aussi les rapporter à leurs déterminants (non seulement leur combinatoire formelle).

\section{La mesure}

Le dernier enjeu stratégique du texte concerne la mesure, terme que nous avons choisi pour englober toutes formes de représentation numérique de l'objet de recherche ainsi que les calculs auxquels il peut donner lieu. Il inscrit le texte dans un entre-deux acceptable pour la communauté scientifique entre le démesuré (ou l'infini) et l'incommensurable..$^{18}$ Il est facile de voir comment les stratégies de mesure se rattachent 
à la validation et à la facticité : impossible de concevoir un objet dans les paradigmes modernes s'il n'a pas au préalable livré sa trace mesurable: "La mesure est alors constitutive du fait, non parce qu'elle le crée, mais parce que, en le stylisant, elle le rend identique pour tous et apte à entrer en combinaison avec d'autres faits ». (Besson, in Hagène et Saliot : 56). Par ailleurs la mesure est une forme d'épure de la logique textuelle : ce qui la fonde et ce vers quoi elle tend. Encore une fois nous constatons que les quatre torons de la tresse de véridiction ne sont que les facettes d'une même réalité.

Les « enjeux » textuels (et non textuels) de la mesure sont trop importants dans ce texte - ils concernent presque la moitié de l'argumentation - pour être traités sommairement, en fin d'article. Ils nécessitent donc une étude particulière, mais il est possible de prévoir que les trames textuelles se déploieront selon trois orientations : 1. La précision qui doit déterminer dans quelles limites les résultats acquis peuvent être tenus pour significatifs (cf. \$2 l'évaluation du « root-mean-square » et l'ensemble de la note 8); 2. Le choix des mesures considérées comme significatives: n'oublions pas, comme Latour l'a montré $(1979,1986)$, que le laboratoire est une usine à produire des « inscriptions littéraires ; » toutes ne sont pas significatives : dans le cadre de notre article, les similarités constatées dans la mise en parallèle des alignements d'acides aminés doivent être soigneusement évaluées et nuancées (cf. $\$ 3$ et 4 ); 3. L'interprétation des résultats aussi bien dans leurs régularités que dans les aberrations constatées fait partie intégrante de la mesure.

\section{Quatre faces de l'article de recherche}

50 L'article scientifique n'est pas un, il est multiple ; il n'est que rarement prédictible dans sa forme sinon à des niveaux relativement superficiels. Il est néanmoins possible d'accéder aux fonctionnements profonds qui fondent ses trames textuelles. Chacun des "enjeux " que j'ai isolés correspond aux quatre faces de l'article de recherche : sociale, thématique, argumentative et formelle. On voit bien à présent combien il est vain de privilégier l'une d'entre elles aux dépens des autres, tant elles sont imbriquées. Même si la linéarité du discours donne l'impression d'une surface lisse, homogène et continue, il ne s'agit que d'une illusion induite par le texte. Il serait donc vain de ne pas tenir compte de la spécificité des enjeux que nous venons de détailler dans l'étude des différentes «traductions » de ces stratégies en langue.

\section{L'inscription linguistique}

51 Il reste à définir, en effet, comment ces orientations stratégiques se réalisent en langue et non l'inverse, c'est à dire comment les formes linguistiques peuvent éclairer certains aspects du DS, ce qui a été l'orientation méthodologique la plus pratiquée jusqu'ici. Devront être envisagées successivement dans une optique de top-down linguistics les différentes inscriptions du DS :

9.1.

52 L'inscription sémiotique, c'est à dire, non seulement la façon dont le contenu informationnel est réparti entre textuel, visuel et formalisé, mais aussi, comment le macrotexte se trouve inscrit dans le texte (c'est l'aspect formel qui sera étudié à ce niveau et non l'orientation stratégique comme précédemment); et, finalement, apprécier le 
rapport entre texte et péri-textes : appendices, notes, bibliographie et surtout titre, et abstract.

\section{2}

L'inscription rhétorique (c'est à ce niveau que l'on retrouve les acquis décisifs des néorhétoriciens) qui consiste à étudier l'influence des différents « genres» de présentation liés aux diverses situations de production et de communication: écrit/oral; communication informelle ou publique; notes de laboratoires ou protocoles d'observations; résumés ou comptes rendus panoramiques, etc. C'est à ce niveau également qu'il faudra s'interroger sur l'incidence du schéma IMRAD sur la structuration des contenus ainsi que sur l'influence des différentes politiques éditoriales sur l'orientation et la rédaction des articles de recherche. J'étendrai cette étude à l'observation de ce que j'appellerai les «gambits» textuels (qui s'apparentent au schéma «move/step» de Swales) parmi lesquels on retrouvera tous les schémas pré-contruits isolés par Trimble et, dans notre article en particulier, le "gambit » de l'analogie dont il faudra étudier tous les aspects remarquablement illustrés par les auteurs de l'article ici analysé. Il va sans dire que notre effort en tant qu'enseignants de langues dans le domaine LANSAD portera sur l'observation et la reproduction de ces "gambits", qui différencient si fortement la production des anglophones de celle des non-natifs.

\section{3.}

Il faudra finalement évoquer l'inscription linguistique des différentes stratégies évoquées plus haut, en évitant de partir d'un comptage statistique des catégories elles-mêmes, qui aurait pour effet d'écraser les valeurs distinctives en discours et de placer, par exemple, dans la même catégorie classificatoire le « may occur » du § 13 à nette valeur épistémique ( " cela peut éventuellement se produire ») avec le « may have important implications » placé immédiatement avant qui fait référence à un degré de certitude posé par les auteurs de l'article et donc non constatable dans la réalité des faits. De même, nous avons montré, incidemment, que les valeurs des passifs peuvent correspondre à des processus effectivement accomplis (du type «Material \& Methods»), à des objets produits ou à des sous-composantes d'objets sans rapport direct avec une quelconque occultation de l'agent qui, de toute façon, n'a pas lieu d'être dans le DS. De même, il est peut-être hasardeux d'assigner, comme le font certains, des valeurs trop définies aux temps utilisés dans, par exemple, l'«abstract» de notre article où l'on trouve, en alternance apparemment déroutante, des présents et des "preterits ": "show structural homology", "The structure suggested that, in general, they adopt... » Des régularités un peu trop générales - comme celle qui voudrait que l'on considère le preterit comme faisant référence à une recherche maintenant dépassée, le «past present » à des recherches associées au thème de l'article qui, quant à lui, ne ferait état de son apport spécifique que dans la forme du présent pourront être nuancées dans le cadre des schémas stratégiques décrits précédemment. Je ne prétends certes pas que ces observations soient fausses mais seulement qu'elles ne peuvent être instituées en tant que lois constitutives du DS : aucune forme linguistique n'a de valeur en soi ; elle ne prend sens, en combinaison avec d'autres, qu'à l'intérieur des stratégies discursives dans lesquelles elle s'insère. 


\section{Conclusion}

Cette étude, malgré son relatif inachèvement, présente tout un corps d'hypothèses et de lignes d'exploration qui demandent à être confirmées et développées dans d'autres domaines de recherche et au contact de sciences beaucoup moins formalisées que l'immunologie. D'ores et déjà, je pense avoir montré les riches possibilités ouvertes par les travaux des sociologues de la science et marqué certaines bornes par rapport à leur recherche en donnant une dimension plus textuelle à leurs concepts. Il est clair que nous n'avons pas affaire à un nouveau champ de recherche, mais à une vision plus nuancée, plus dynamique et plus adaptable des trames textuelles qui constituent l'article de recherche. Même si j'ai insisté sur l'importance qu'il y avait à dépasser la surface lisse et monolithique de la structure IMRAD pour envisager les enjeux sociocognitifs sousjacents, il me semble possible d'intégrer les avancées décisives accomplies dans l'optique néo-rhétoricienne. L'article de recherche tel que nous le représentons apparaît à la fois plus complexe et plus fascinant, peut-être aussi plus abordable et, une fois les premières résistances passées, plus susceptible d'entretenir une riche collaboration avec ses producteurs. L'article de recherche est la recherche d'un équilibre précaire dans un univers discursif en constante situation de déstabilisation. La stratégie de véridiction est la trace complexe mais tangible de cet équilibre.

\section{BIBLIOGRAPHIE}

Barnes, B. 1974. Scientific Knowledge and Sociological Theory. Londres : Routledge and Kegan Paul.

Barnes, B. and D. Edge. 1982. Science in Context. Readings in the Sociology of Science. Cambridge, MA : M.I.T. Press.

Callon, M. 1994. La science et les réseaux. Paris : La Découverte.

Coulthard, M. (dir.). 1994. Advances in Written Text Analysis. Londres/New York : Routledge.

Dudley-Evans, Tony. 1994. «Academic text: The importance of the use and comprehension of hedges ». ASp 5-6, 131-139.

Feyerabend, P. 1988. Against Method (Revised Edition). Londres : Verso Books (First published : New Left Books, 1975).

Fuller, S. et al. 1989. The Cognitive Turn. Sociological and Psychological Perspectives on Science. Dordrecht : Kluwer Academic Publishers.

Gilbert, G. N. and M. Mulkay. 1984. Opening Pandora's Box: A Sociological Analysis of Scientists' Discourse. Cambridge : Cambridge University Press.

Gusfield, J. 1976. « The literary rhetoric of science: Comedy and pathos in drinking driver research ». American Sociological Review 41/2, 16-33.

Hagène, B. et P. Saliot. 1995. Mesures et démesure. Paris : Cité des Sciences et de l'Industrie. 
Halliday, M. A. K. 1994. « The Construction of science and value in the grammar of scientific discourse, with reference to Charles Darwin's The Origin of Species ». In Coulthard, M., 136-156. Henderson, W., T. Dudley-Evans and R. Backhouse. 1993. Ecomomics and Language. Londres : Routledge.

Knorr, K., R. Krohn \& R. Whitley (dir.). 1980. The Social Process of Scientific Investigation, 4 Reidel Pub. Co.

Knorr-Cetina, K. 1981. The Manufacture of Knowledge: An Essay on the Constructivist and Contextual Nature of Science. Oxford : Pergamon.

Kuhn, T. S. 1983. La structure des révolutions scientifiques. Paris : Champs, Flammarion.

Latour, B. 1980. « Is it possible to reconstruct the research process? ». In Knorr-Cetina, K., The Manufacture of Knowledge: An Essay on the Constructivist and Contextual Nature of Science. Oxford : Pergamon.

Latour, B. 1981. The Manufacture of Knowledge. An Essay on the Constructivist and Contextual Nature of Science. Oxford : Pergamon Press.

Latour, B. 1987. Science as Action. Cambridge, MA : Harvard University Press.

Latour, B. 1990. « Drawing things together ». In Lynch, M \& S.Woolgar (dir.), Representation in Scientific Practice. Cambridge, MA : M.I.T Press.

Latour, B. 1993. La clef de Berlin. Paris : La Découverte.

Latour, B. 1994. Nous n'avons jamais été modernes. Paris : La Découverte.

Latour, B. \& P. Fabbri. 1977. « Pouvoir et devoir dans un article de science exacte ». Actes de la recherche en sciences sociales $13,81-95$.

Latour, B. \& S. Woolgar 1986 [1979]. Laboratory Life: The Construction of Scientific Facts. Princeton, NJ : Princeton University Press (Sage 1979).

Lynch, M. 1985. Art and Artifact in Laboratory Science. A Study of Shop Work and Shop Talk in a Research Laboratory. Londres : Routledge \& Kegan Paul.

Lynch, M. \& S. Woolgar (dir.). 1990. Representation in Scientific Practice. Cambridge, MA : M.I.T Press.

Miller, T. 1993, « The Introduction to the research article from a discourse perspective ». ASp 2, 55-68.

Mulkay, M. 1979. Science and the Sociology of Knowledge. Londres : Allen \& Unwin.

Myers, G.1985. The Word and the World: Explorations in the Form of Sociological Analysis. Londres : George Allen \& Unwin.

Myers, G. 1990. Writing Biology. Texts in the Social Construction of Scientific Knowledge. Madison : University of Wisconsin Press.

Myers, G. 1991. « Scientific speculation and literary style in a molecular genetics article ». Science in Context 4/2, 324-341.

Myers, G. 1994. « Narratives of science and nature in popularizing molecular genetics ». In Coulthard, M. (dir.), Advances in Written Text Analysis. Londres/New York : Routledge, 179-218.

Sager, J. C., D. Dunkworth and P. F. McDonald. 1990. English Special Languages. Principles and Practice in Science and Technology. Wiesbaden : Oscar Brandsletter Verlag KG. 
Salager-Meyer, F. 1994. « Hedges and textual communicative function in medical English written discourse ». English for Specific Purposes 13/2, 149-170.

Salager-Meyer, F. 1995. «I think that perhaps you should: A study of hedges in written scientific discourse ». TESOL-France 2/2, 127-144.

Selinker L., E. Tarone and V. Hanzeli (dir.). 1981. English for Academic and Technical Purposes. Rowley, MA : Newbury House.

Swales, J. M. 1981. Aspects of Article Introductions. Birmingham : University of Aston.

Swales, J. M. 1990. Genre Analysis. English in Academic and Research Settings. Cambridge : Cambridge University Press.

Trimble, L. 1985. English For Science and Technology. Cambridge : Cambridge University Press.

Widdowson, H. G. 1983. Learning Purpose and Language Use. Oxford : Oxford University Press.

Woolgar, S. 1981. « Discovery: Logic and sequence in a scientific text ». In Knorr-Cetina, K., The Manufacture of Knowledge: An Essay on the Constructivist and Contextual Nature of Science. Oxford : Pergamon, 240-263.

Woolgar, S. 1989. « Representation, cognition and Self: What hope for an integration of psychology and sociology? ». In Fuller, Steve et al. (dir.), The Cognitive Turn. Sociological and Psychological Perspectives on Science. Dordrecht : Kluwer Academic Publishers.

\section{NOTES}

1. Ce texte reprend en substance des notes de cours distribuées (et malheureusement trop tôt diffusées dans le cadre du GERAS) aux étudiants du DEA multi-sceaux de Bordeaux, Montpellier, Toulouse et Paris 4. Les raisons qui m'ont poussé à envisager cette publication, que certains pourraient juger impertinente, sont au nombre de trois. 1) Une grande partie des informations d'arrière-plan nécessaires pour la comprendre avait été donnée en cours et donc faisait défaut. 2) L'article a subi une refonte considérable dans l'axe de la recherche que je poursuis sur le DS. 3) Le "papier» de travail était un papier gras entaché d'erreurs typographiques pour ne citer que celles-là... Les paragraphes de l'article ont été numérotés dans l'ordre pour faciliter les références.

2. «Crystal Structure of the B Chain of a T Cell Antigen Receptor ». Science 267, 31 March 1995, p. 1984-87.

3. Ma bibliographie donne un échantillon assez représentatif de leurs travaux.

4. Les notions que nous avons isolées se rapportent aux travaux, de notre point de vue, les plus aboutis et les plus influents, à savoir, ceux de Bruno Latour. Mais, je suis conscient, en raison du propos proprement linguistique de cette étude et de ses limites physiques, d'avoir opéré une réduction drastique de ses concepts, de l'évolution de sa pensée et, plus généralement de la complexité de la réflexion de la recherche en sociologie des sciences.

5. Notamment les chercheurs gravitant autour de Michael Mulkay.

6. Cf., tout particulièrement, l'article de Latour et Fabbri (1977).

7. Cf. la note 37.

8. Cf. en plus de l'encart au bas de la première page, les notes 6, 7 et 8 .

9. Cf. sur ces points les analyses décisives de Myers et Latour.

10. Cet aspect de l'article scientifique correspond plus ou moins au "degré de technicité » dans d'autres approches, mais l'on voit comment ce concept bien connu est posé ici de façon dynamique et donc plus productive pour la recherche. 
11. Il est possible d'étendre la validité de cette observation à l'ensemble des "Introductions » dont le rôle est, si l'on garde en mémoire l'analogie, de faciliter la « repousse» des textes et d'éviter les phénomènes de rejet.

12. L'utilisation des crochets signifie qu'il s'agit d'une relation pré-assertive.

13. Ce terme correspond en gros aux « hedges » de Salager et Dudley-Evans, mais a l'avantage de se rattacher à une catégorie linguistique bien connue, et donc de produire des classifications un peu plus fiables que celles présentées jusqu'ici - la plus satisfaisante étant celle de Salager (1994,1995).

14. Là aussi, aussi nous faisons une distinction, comme pour le couple-vérité/véridiction, avec ce qui est habituellement perçu comme « réalité, » en risquant l'interférence avec ce qui serait conçu comme factice ou véridique.

15. Cf. Latour sur la nature «littéraire» des informations produites par les instruments de laboratoire (Latour 1986 [1979] : 45 et seq.).

16. Cet article concerne essentiellement la structure des TCR, mais l'on perçoit de timides, mais fermes jalons du côté de leur fonctionnement présumé... qui relie cette recherche au terrain stratégiquement brûlant de la recherche sur le SIDA.

17. Doute qui s'exprime dans les commentaires éditoriaux de ce numéro de Science.

18. Cf. L'excellent ouvrage de Bernard Hagène et Pierre Saliot, Mesures et démesure (1995).

\section{RÉSUMÉS}

Cet article propose, à la suite des travaux des sociologues de la science, un nouveau corps d'hypothèses de nature à rendre compte des fondements sociocognitifs du discours scientifique. L'analyse de la stratégie de véridiction est esquissée à propos d'un article de Science dans le domaine immunologique.

In the wake of recent developments in the sociology of science this article proposes a new body of concepts to account for the socio-cognitive underpinnings of scientific discourse. There follows an analysis of the strategy of veridiction in an article from Science on immunology.

\section{INDEX}

Mots-clés : discours scientifique, macrotexte, sociologie de la science, stratégie textuelle, véridiction

Keywords : macrotext, scientific discourse, sociology of science, textual strategy, veridiction

\section{AUTEUR}

JACKY MARTIN

Professeur à l'Université Paul-Valéry Montpellier 3. jacky.r.martin@neuf.fr 